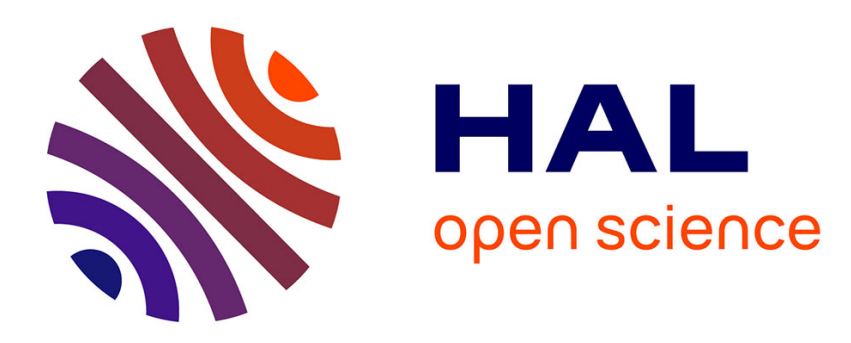

\title{
De l'interaction avant toute chose... Temps verbaux et relation de progression narrative
}

Jacques Bres

\section{To cite this version:}

Jacques Bres. De l'interaction avant toute chose... Temps verbaux et relation de progression narrative. Cahiers Chronos, 2009, 21, pp.45-64. hal-00329885

\section{HAL Id: hal-00329885 \\ https://hal.science/hal-00329885}

Submitted on 3 Jul 2018

HAL is a multi-disciplinary open access archive for the deposit and dissemination of scientific research documents, whether they are published or not. The documents may come from teaching and research institutions in France or abroad, or from public or private research centers.
L'archive ouverte pluridisciplinaire HAL, est destinée au dépôt et à la diffusion de documents scientifiques de niveau recherche, publiés ou non, émanant des établissements d'enseignement et de recherche français ou étrangers, des laboratoires publics ou privés. 


\title{
De l'interaction avant toute chose... Temps verbaux et relation de progression narrative
}

\author{
Jacques Bres \\ Praxiling, UMR 5267 CNRS-Montpellier III
}

Voilà dix ans, je commençais, presque par hasard, à fréquenter les temps verbaux, mi-amusé mi-séduit par le maquillage de l'imparfait dans son emploi narratif (Bres 1998). Rapidement la passade s'installa dans la durée, je devins un amoureux du temps sous toutes ses formes verbales. Je m'autorise de cette longue fréquentation pour pointer quelques écueils que j'ai rencontrés. Je présente ensuite rapidement l'hypothèse de travail que j'ai développée afin de la tester sur l'analyse de la relation de progression narrative.

\section{De quelques écueils}

Rétrospectivement et sans vouloir aucunement être donneur de leçon, il me semble que l'analyse du temps verbal est toujours au risque de buter sur ce que j'appréhende comme des difficultés, consistant, pour le dire de façon imagée, à prendre les temps pour des îles, l'arbre pour la forêt ou l'ombre pour la proie.

\subsection{Prendre les temps pour des îles}

Ce premier écueil consiste à ne s'intéresser qu'à une forme verbale, en oubli du système que forme leur ensemble, ce qui est du même coup oublier Saussure et la valeur différentielle du signe. De la sorte sera p. ex. travaillé le passé simple sans le mettre en rapport avec le passé antérieur, l'imparfait ou le passé composé, avec lesquels il entretient pourtant des relations de détermination réciproque. Comme si un phonologue étudiait [p] sans se préoccuper ni de $[\mathrm{b}]$ ni de $[\mathrm{m}]$, ni du micro-système d'occlusives bilabiales que forment ces phonèmes par rapport au micro-système des occlusives dentales... Les temps ne sont pas des îles; ils sont plutôt, si l'on veut poursuivre la métaphore, un archipel dont les inter-relations ne sauraient être négligées car elles sont pleinement structurantes de leur valeur.

\subsection{Prendre l'arbre pour la forêt}

Le deuxième écueil consiste à prendre un effet de sens ou un emploi, majoritaire en discours, pour la valeur en langue du temps verbal. Sous l'influence de la pragmatique et de la linguistique textuelle, de nombreuses 
études ont tenté de définir un temps verbal par tel ou tel de ses comportements textuels, ce qui est confondre le plan de la langue et celui du discours. Typiques p. ex de cette démarche sont les explications qui ont été avancées pour le passé simple et l'imparfait en termes (i) de plans (ils situeraient le procès respectivement dans le premier plan et dans l'arrièreplan); (ii) de relations temporelles (ils introduiraient, par rapport au précédent procès, une relation de progression pour le premier, de simultanéité pour le second) ; (iii) de point de vue (ils actualiseraient le procès objectivement pour le premier, subjectivement pour le second). Si tel est souvent le cas, ce ne l'est pas toujours : l'analyse bute alors sur des exceptions, et des trésors d'ingéniosité sont déployés pour tenter de blanchir le cygne noir.

\subsection{Prendre l'ombre pour la proie}

Le troisième écueil consiste à attribuer par imputation abusive telle ou telle valeur au temps verbal, alors qu'il ne s'agit que d'un effet de sens, bien réel au niveau du discours, produit par l'interaction de la valeur en langue de ce temps avec son contexte, lors de l'actualisation. Exemple type de cette démarche, l'analyse de l'imparfait comme développant une valeur modale contrefactuelle dans un tour comme :

(1) Le père, sans s'arrêter de planter les piquets, de tasser ses pierres, grognait : - Quinze jours de plus, et la vigne davallait à la rivière ; après, il faut cent ans pour la remonter! (Chabrol, Les Fous de Dieu)

On comprend que la vigne n'a pas «davallé » ('descendre' en occitan) jusqu'à la rivière... Et les grammaires voient là un emploi modal de l'imparfait dans la mesure où ce temps aurait la vertu de pouvoir signifier ici ce qui n'a pas eu lieu mais aurait pu se produire.

Une autre analyse est possible, que ce soit dans les cadres de l'anaphore méronomique ou dans ceux de l'approche aspectuo-temporelle : la contrefactualité, bien réelle, procède de la structure syntaxique de l'énoncé, et de son interaction avec le cotexte; l'imparfait, du fait de son fonctionnement méronomique (Berthonneau \& Kleiber 2003) ou de sa structure aspectuelle (Bres 2006), peut se conjoindre à cet effet de sens.

Prendre l'ombre pour la proie, c'est voir des imparfaits narratif, préludique, hypocoristique, contrefactuel, forain, etc... là où il n'y a qu'un seul et même imparfait qui, en interaction avec des contextes différents, entre, à titre d'ingrédient, dans la production des effets de sens - narratif, préludique, hypocoristique, contrefactuel, forain - repérables au niveau de l'énoncé. 


\section{Les temps verbaux : de la langue au discours}

Pour rendre compte des fonctionnements en discours des temps verbaux comme de leur structuration systémique en langue, on s'appuie sur les trois hypothèses suivantes :

(i) hypothèse systémique et aspectuo-temporelle : les temps verbaux forment un système en langue, qui se construit sur les deux paramètres du temps et de l'aspect (entre autres Guillaume 1929, Wilmet 1997, Barceló \& Bres 2006). Les temps verbaux donnent une instruction temporelle qui permet de situer le procès qu'ils actualisent dans une des trois époques (passée, présente, future) ${ }^{1}$. Aspectuellement, le système du français se construit sur les trois éléments de la tension, de l'incidence et de la prospection.

- La tension permet de distinguer les formes simples (soit [+ tension]), qui représentent le temps interne dans l'espace qui va de sa borne initiale à sa borne terminale ; des formes composées (soit [+ extension]), qui le saisissent à partir ou au-delà de sa borne terminale. Nous ne prenons pas en compte ici les formes surcomposées.

- L'incidence permet de distinguer (i) les formes qui représentent le temps interne en seul accomplissement, de sa borne initiale à sa borne finale (passé simple), ou à partir de sa borne finale (passé antérieur), (soit [+ incidence]); (ii) les formes qui représentent le temps interne en conversion de l'accomplissement en accompli, en un point situé au-delà de sa borne initiale et en-deçà de sa borne terminale (imparfait), ou au-delà de sa borne terminale (plus-que-parfait), (soit [- incidence]) ; et (iii) les formes qui sont neutres visà-vis de cette catégorie (présent, futur, conditionnel), (soit [ \pm incidence]).

- La prospection permet de décrire les temps formés sur la grammaticalisation de aller (il va pleuvoir, il allait pleuvoir) ${ }^{2}$.

1 Ajoutons que l'instruction [+ futur] «s'allège » en [+ ultériorité] lorsqu'elle se combine avec l'instruction [+ passé] dans la description du conditionnel (cf. infra tableau 1).

2 On ne pose pas, symétriquement à la prospection construite sur aller, la rétrospection construite sur venir (il vient / venait de pleuvoir) dans la mesure où venir est moins grammaticalisé que aller: ses emplois se cantonnent à la récence et ne concurrencent vraiment ni le passé composé ni le plus-que-parfait. 
instruction temporelle instructions aspectuelles

PS :

il plut

[+ passé $]$

[+ tens. $]$ [+ incid. $]$

PA :

il eut plu [+ passé $]$

IMP :

il pleuvait [+ passé

[+ passé]

[+ ext. $\quad$ [+ incid. $]$

PqP :

il avait plu [+ passé $]$

[+ tens. $] \quad$ [- incid. $]$

FS :

il pleuvra [+ futur]

[+ ext.] [- incid.]

FA :

il aura plu $\quad[+$ futur

Cd PR :

il pleuvrait [+ passé] [+ ultérieur]

[+ tens. $]$ [ \pm incid. $]$

Cd. P :

[+ passé $\quad$ [+ ultérieur]

[+ ext. $]$ [ \pm incid. $]$

il aurait plu

[+ tens.] [ \pm incid. $]$

PR :

il pleut

[+ neutre]

[+ ext.] [ \pm incid.]

PC :

il a plu

[+ neutre]

[+ tens.] [ \pm incid.]

PR prosp :

[+ ext.] [ \pm incid. $]$

il va pleuvoir

PR prosp. ext. :

il va avoir plu

[+ neutre]

[+ tens.] [t incid.]

IMP prosp :

il allait pleuvoir

IMP prosp.ext. :

il allait avoir plu [+ passé $] \quad$ [+ prosp. $] \quad$ [+ ext. $] \quad[-$ incid. $]$

Cette description unifiée des temps verbaux permet de les décrire comme des combinaisons différentes des mêmes éléments : le passé simple p. ex. partage avec l'imparfait l'élément de la tension mais s'en différencie par l'incidence ; il partage avec le passé antérieur l'élément de l'incidence mais s'en différencie par la tension.

(ii) hypothèse interactionniste : on passe de la langue au discours non par une solution de continuité, mais par l'opération cognitive infraconsciente de l'actualisation au cours de laquelle les instructions de la valeur en langue du temps verbal entrent en interaction avec les différentes valeurs des différents morphèmes du cotexte, et avec le contexte, pour produire, résultativement, tel ou tel effet de sens observable en discours. À des fins d'analyse, on 
distinguera entre la demande du co(n)texte et l'offre du temps verbal, qui interagissent selon trois modalités : l'interaction peut être concordante (infra 3.1.), tendanciellement discordante (infra 3.2.), frontalement discordante.

(iii) Hypothèse monosémiste : dans la diversité des sens produits, le temps verbal donne toujours les mêmes instructions, celles qui définissent sa valeur en langue. La pluralité observable au niveau discursif est le résultat de l'interaction des mêmes instructions du temps verbal avec des éléments co(n)textuels différents. On s'oppose par là aux approches polysémistes qui rendent compte de ladite pluralité en recourant à la notion de variation quantitative (Guillaume 1929) ou à celle de déformation (Gosselin 1996). Pour reprendre l'ex. de l'imparfait mentionné supra en 1.3., c'est avec ses mêmes instructions: [+ passé], [+ tension], [- incidence] qu'il intervient comme ingrédient dans la production des effets de sens narratif, hypocoristique, contrefactuel, etc...

Je me propose de tester le rendement de cette approche systémique et aspectuo-temporelle de la valeur en langue des temps verbaux, et de cette analyse des effets de sens comme produits en discours par l'interaction de ladite même valeur avec des contextes différents, sur un objet bien précis : la relation de progression narrative.

\section{Temps verbaux et relation de progression narrative}

Les relations temporelles entre deux procès $[x]$ et $[y]$ référant à deux événements peuvent être de simultanéité $([x=y])$, de progression $([x<y])$, de régression $([x>y])$, d'inclusion $([x \subset y])$, etc. (cf . notamment Lascarides \& Asher 1993, Asher \& al. 1995).

Quel rapport entre temps verbaux et relations temporelles? On fait l'hypothèse que les instructions données par les temps verbaux et les relations temporelles entre les événements auxquels référent les procès sont des faits autonomes qui relèvent de deux ordres différents : celui de la langue pour les premières, celui du discours pour les secondes. Autonomie ne veut cependant pas dire indépendance : lors de la mise en discours, dans le temps d'actualisation, le temps verbal interagit avec le contexte, notamment avec les relations d'ordre temporel.

Les relations temporelles sont construites par le contexte, à savoir, pour le dire rapidement, nos connaissances du monde et la situation d'interaction ; par le cotexte (notamment les conjonctions et circonstants temporels, la syntaxe, les types de procès); et par l'interaction de ces éléments avec les instructions aspectuelles du temps verbal, mais en rien directement par le temps verbal lui-même. En fonction des instructions qu'il offre, il a plus ou moins d'affinité ou d'antipathie avec la demande de telle ou telle relation temporelle : il participera activement à la production de celle-ci, s'associera 
simplement à celle-là, fera quelques difficultés avec cette autre, ne pourra se conjoindre enfin à cette quatrième...

On s'intéressera dans cet article au rapport entre les temps verbaux de l'indicatif et la relation de progression, et plus précisément sa réalisation en textualité narrative.

La relation de progression définit des genres de discours aussi différents que la recette de cuisine, l'indication d'itinéraire, la description d'action, le conte ou le récit conversationnel, etc. Seuls les deux derniers relèvent de la textualité narrative.

La textualité narrative est habituellement définie comme enchaînement de propositions narratives (Labov 1972/1978) dont l'ordre tend à (re)produire l'ordre des événements (du premier plan), ce qui implique que, d'une proposition à l'autre, le temps (raconté) auquel il est fait référence progresse :

(2) Mona s'assit sur le bord du divan en faisant un petit soupir de fatigue, puis, de nouveau, de ce geste du menton qu'elle avait, rejeta ses cheveux en arrière et leva vers Grange ses yeux et sa bouche, avec un étirement de plante qui prend le soleil (Gracq, Un Balcon en forêt)

Dans ce fragment narratif, on a trois propositions narratives, dont l'ordre successif dans le texte (re)produit l'ordre progressif des événements. Soit en mettant les procès à l'infinitif : [s'asseoir < rejeter < lever]. Cognitivement, la relation de progression entre plusieurs procès consiste à parcourir le temps interne du premier procès de sa borne initiale $\mathrm{A}$ à sa borne terminale $\mathrm{B}$, et à passer de celle-ci à la borne initiale $\mathrm{C}$ du second procès ; à parcourir le temps interne de ce second procès de sa borne initiale à sa borne terminale $\mathrm{D}$, et à passer de celle-ci à la borne initiale $\mathrm{E}$ du troisième procès, et ainsi de suite. Soit :

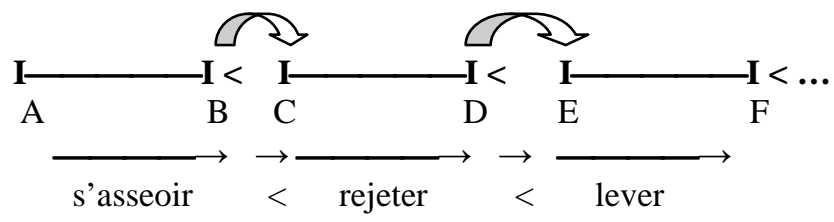

Cette relation demande donc que le temps interne des procès soit actualisé (i) dans sa tension, c'est-à-dire dans sa réalisation et non au-delà ; et (ii) en incidence, à savoir comme parcours de la borne initiale à la borne terminale. Soulignons l'importance du marquage de la borne terminale : la progression d'un procès à l'autre se fait de la borne terminale du premier à la borne initiale du second, etc.; et l'atteinte de la borne terminale pose que le procès a bien eu lieu. 
La relation de progression concerne donc la dimension aspectuelle du temps verbal; elle se complète, en textualité narrative, de la dimension temporelle : l'événement mis en récit est censé s'être passé (réellement ou fictivement), il doit précéder sa narration, ce qui demande que le temps externe des procès relève de l'époque passée ou soit compatible avec elle, et tend à exclure l'époque future.

En fonction de leur offre aspectuo-temporelle, les temps verbaux seront en interaction parfaitement concordante (3.1.), ou en partie discordante (3.2. et 3.3.) avec la relation de progression narrative.

\subsection{Interaction concordante : le passé simple et le présent}

Ces deux temps s'accordent avec la demande de la relation de progression narrative.

\subsubsection{Passé simple : [+ passé], [+ tension], [+ incidence]}

Reprenons l'ex. (1). Le passé simple demande de situer l'événement auquel fait référence le procès dans le passé, et représente le temps interne (i) en tension, et (ii) de sa borne initiale jusqu'à sa borne terminale; il s'accorde parfaitement à la demande de la relation de progression narrative :

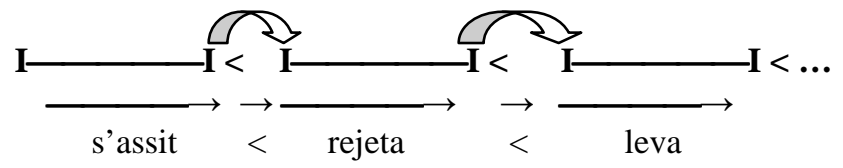

Voilà donc pourquoi le passé simple est le temps narratif par excellence, même s'il ne donne pas lui-même l'instruction [+ progression]. Ce qui rend compte du fait que, s'il est dans les textes massivement associé à ce type de relation, il peut entrer dans d'autres relations discursives, comme la simultanéité ou même la régression. La concurrence forte (Labeau 2007) que lui font le passé composé et le présent en textualité narrative tient à des raisons énonciatives (Benveniste 1959 / 1966).

\subsubsection{Présent : [+ neutre], [+ tension], [ \pm incidence]}

En ne situant pas en lui-même le procès dans une époque (instruction [+ neutre]), le présent permet d'actualiser des faits par ailleurs posés comme passés. En représentant le temps interne dans sa tension et de façon neutre au regard de l'incidence, il répond à la demande cotextuelle de progression. C'est la raison pour laquelle ce temps est très souvent employé, notamment 
en récit oral conversationnel (Carruthers 2005) pour actualiser les actions du premier plan :

(3) Conversation familiale. Un chasseur raconte, au cours du repas :

/ je monte ici lundi dernier / dans une heure je tire trois lièvres / j'en tue point / l'après-midi j'en manque un autre / ça fait quatre [...]

\subsection{Interaction latéralement discordante: imparfait, passé antérieur, passé composé, plus-que-parfait}

Ces quatre temps, s'ils s'accordent avec la demande temporelle [+ passé] de la textualité narrative, présentent une discordance avec la demande aspectuelle, qui peut porter sur un des deux éléments requis par la progression : la tension, l'incidence ; ou sur les deux éléments à la fois.

\subsubsection{Discordance portant sur l'incidence : l'imparfait}

L'imparfait, qui donne les instructions [+ tension], [- incidence], est en accord avec la demande de tension de la relation de progression, mais en désaccord avec la demande d'incidence. On a donné à ce tour le nom d'imparfait narratif, ou de rupture, que l'on trouve dans l'écrit littéraire et journalistique, mais également dans l'oral conversationnel :

on a bu du vin blanc / plusieurs bouteilles et la semaine d'après il nous faisait un crise de goutte que je te raconte pas (conversation entre amis)

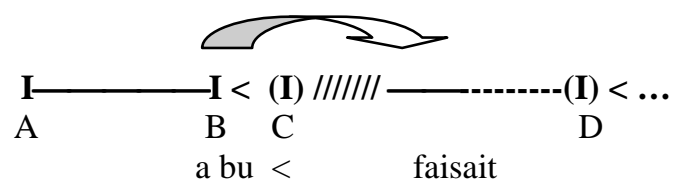

Dans la relation de progression, l'imparfait, en ne donnant pas à voir la borne initiale $\mathrm{C}$ attendue, impose un saut cognitif par-dessus ladite borne. En ne conduisant pas la représentation du temps interne impliqué par le procès jusqu'à son terme $\mathrm{D}$, il maintient le point de référence en deçà de ce qui est également attendu... Cette légère discordance se résout par la production contextuelle de nombreux effets stylistiques, dont cette impression complexe dans laquelle se conjuguent les contraires de l'accélération (saut par-dessus la borne initiale) et de la décélération (arrêt avant la borne terminale). 
3.2.2. Discordance portant sur la tension. Le passé composé, le passé antérieur

1. Le passé composé s'accorde avec la demande d'incidence, formulée par la relation de progression, du fait de sa neutralité (son instruction [ $[ \pm$ incidence]) ; mais entre en désaccord avec la demande de tension : comme toute forme composée, il est d'instruction [+ extension]. Et pourtant, depuis le Moyen-Age, le passé composé concurrence le passé simple en contexte narratif. On sait qu'il l'a totalement remplacé dans le plan d'énonciation du discours (Benveniste 1959 / 1966). Décrivons sommairement son fonctionnement :

(...) y a deux gendarmes qui sont venus ils nous ont dit «Là vous passez pas » / on est repartis (interview Corpus Ladrecht)

En interaction avec le cotexte narratif, le passé composé produit l'effet de sens 'événement passé' : comme le passé simple, il saisit le temps interne des procès venir et dire globalement (ce qui rend compte de ce qu'il puisse le concurrencer) ; mais alors que le passé simple opèrerait cette saisie à partir de la borne initiale (A pour venir, $\mathrm{C}$ pour dire, $\mathrm{E}$ pour repartir), le passé composé le fait, comme toute forme composée, à partir de la borne terminale (B pour venir, $\mathrm{D}$ pour dire, $\mathrm{F}$ pour repartir). Ce qui implique que, dans l'actualisation de la progression [sont venus < ont dit < est repartis], l'énonciateur saute cognitivement la partie tensive C-D de dire, pour la saisir rétrospectivement à partir de la borne terminale $\mathrm{D}$; puis saute la partie tensive de repartir pour la saisir rétrospectivement à partir de la borne terminale $\mathrm{F}$ :

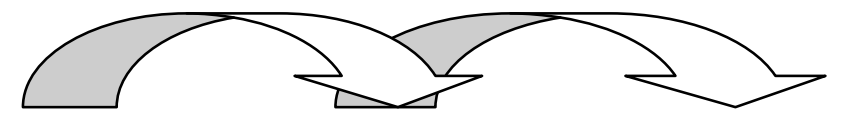

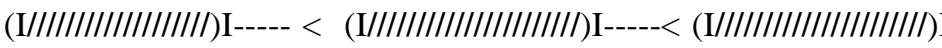
$--$
A
B C
sont venus
D E
$\mathrm{F}$
est repartis

De nombreux auteurs ont noté, sans vraiment l'expliquer, qu'avec le passé composé se perdait la fluidité du récit, que chaque procès apparaissait non pas comme ouvert sur le suivant mais comme refermé sur lui-même. Rappelons que Sartre parlait de la phrase au passé composé de L'Étranger comme d'une île: appréhension imagée fort pertinente du peu d'affinité intrinsèque du passé composé pour la relation de progression... 
C'est parce que le passé composé saisit globalement le temps interne du procès à partir de sa borne terminale qu'il entre en interaction partiellement dissonante avec la demande cotextuelle de la relation de progression de le représenter à partir de sa borne initiale : s'il permet de raconter, il n'est pas un temps narratif parfait. Ce qui se traduit notamment par le fait suivant : le passé composé, alors qu'il concurrence le passé simple depuis des siècles, ne parvient pas vraiment à l'éliminer. Le moindre récit écrit de fait divers dans la presse fait le plus souvent alterner, pour actualiser les relations de progression du premier plan, passé composé, présent, passé simple et imparfait « narratif ».

2. De façon similaire, le passé antérieur, de par ses instructions [+ extension], [+ incidence], est en accord avec la demande d'incidence de la relation de progression, mais en désaccord avec la demande de tension. Il saisit le temps interne du procès non à partir de la borne initiale $(\mathrm{C})$ mais à partir de la borne terminale (D) :

Il continua l'aventure commencée par Mme Forestier [...]. En une heure, il eut terminé une chronique qui ressemblait à un chaos de folies, et il la porta, avec assurance, à La Vie Française. (Maupassant, Bel-Ami)

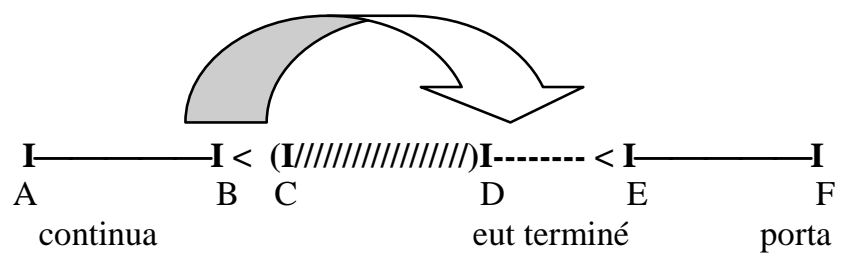

Cependant, le passé antérieur, pour des raisons que nous n'expliquons pas ici, est actuellement une forme fortement contrainte dans ses emplois en discours : en indépendante ou principale, il ne peut guère être employé qu'en appui sur un adverbe signifiant la rapidité (rapidement, bientôt), un circonstant de durée globale (en $x$ temps) ou de datation (à $x$ heure), entre deux passés simples, comme dans (6). Son usage est donc très sporadique, à la différence de ce qui était le cas en ancien français. Comme le passé composé, il induit cognitivement dans l'enchaînement de la progression [continua < eut terminé], le saut de la partie tensive du procès qu'il actualise (terminer). Stylistiquement, il pourra être associé, avec certains marqueurs, à la production cotextuelle d'un effet de sens de rapidité :

(7) La cigogne au long bec n'en put attraper miette, / Et le drôle eut lapé le tout en un moment. (La Fontaine, Le renard et la Cigogne) 
En saisissant le procès à partir de sa borne terminale alors que la demande de la relation de progression était de le saisir à partir de sa borne initiale, le passé antérieur, en interaction avec le SP en un moment, semble dire que l'action s'est faite si vite qu'elle n'a pu guère être saisie que déjà réalisée.

\subsubsection{Discordance portant sur la tension et sur l'incidence. Le plus-que- parfait}

Le plus-que-parfait, de par ses instructions [+ extension], [- incidence], est doublement en désaccord avec la relation de progression. Et pourtant, on le trouve en contexte narratif, dans différents emplois textuels (Bres 2007). Analysons un seul d'entre eux :

$$
\begin{aligned}
& \text { Et, tout d'un coup, comme ils passaient près d'un talus gazonné, et qu'elle } \\
& \text { l'y entraînait, s'allongeant, le besoin monstrueux le reprit, il chercha parmi } \\
& \text { l'herbe une arme, une pierre, pour lui en écraser la tête. D'une secousse, il } \\
& \text { s'était relevé, et il fuyait déjà, éperdu. (Zola, La Bête humaine) }
\end{aligned}
$$

Soit la progression [chercha < s'était levé]. Enchâner, selon la relation de progression, un passé simple et un plus-que-parfait, c'est comme pour le passé antérieur, faire l'ellipse du temps interne du second procès, pour le saisir même pas sur sa borne terminale mais au-delà de sa borne terminale (du fait de l'instruction [- incidence]) :

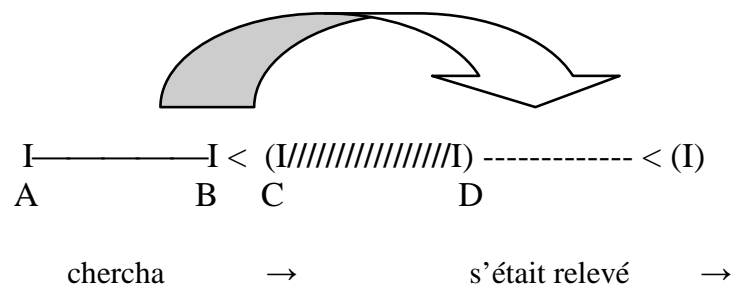

Dans ce type d'emploi, le plus-que-parfait pourra être associé, comme le passé antérieur de (7), à la production cotextuelle d'un effet de sens de rapidité. Soulignons que le plus-que-parfait n'a pas les mêmes contraintes d'emploi que le passé antérieur (cf. supra 3.2.2.), qui dans (8) serait impossible :

(8') [...] il chercha parmi l'herbe une arme, une pierre, pour lui en écraser la tête. D'une secousse, il *se fut relevé, et il fuyait déjà, éperdu.

Comparons pour finir imparfait et plus-que-parfait en cotexte narratif : le plus-que-parfait pourrait sembler plus dissonant que l'imparfait dans la mesure où, alors que celui-ci est en désaccord avec la demande de la relation 
de progression sur la seule catégorie de l'incidence, celui-là ne la satisfait pas doublement : sur l'incidence et sur la tension. Ce qui se manifeste par le fait que l'ellipse du temps interne est de plus d'importance dans l'enchaînement [passé simple < plus-que-parfait] que dans l'enchaînement [passé simple < imparfait]. Et pourtant grammairiens et linguistes parlent d'imparfait narratif mais pas du plus-que-parfait narratif. Serait-ce que, tout à leur extrême sollicitude à l'égard de l'imparfait, ils en auraient oublié un fait plus significatif touchant au plus-que-parfait ? Certainement pas : intuitivement, la dissonance du plus-que-parfait en (8) apparaît moins forte que celle de l'imparfait en (4). Pour rendre compte de cette apparente contradiction entre le fait que l'offre du plus-que-parfait contrevient doublement à la demande aspectuelle de la relation de progression mais que la dissonance qu'il produit apparaît comme moins forte que celle procédant de l'imparfait, qui pourtant n'est en désaccord avec elle sur un seul point, il nous faut revenir sur un élément de ladite demande que nous n'avons jusqu'à présent pas vraiment pris en compte : la demande d'atteinte de la borne terminale du procès. Le passé simple, de par son instruction [+ incidence], y parvient au terme de son parcours du temps interne; le plus-que-parfait la présuppose atteinte puisqu'il se construit au-delà de cette borne ; alors que l'imparfait de par son instruction [- incidence] ne conduit pas la représentation jusqu'à elle. Et c'est cette position différente par rapport à l'atteinte de la borne terminale qui rend compte de ce que le plus-que-parfait, dans la relation de progression narrative, apparaît comme une forme moins marquée que l'imparfait; et que son emploi en contexte narratif se développe actuellement (Majumdar et Morris 1980).

C'est ce même élément qui fait que le passé composé a pu devenir un temps narratif - même imparfait - de base du français : présupposant la borne terminale atteinte, il permet à la relation de progression de se développer, même si c'est d'une façon moins fluide qu'avec le passé simple ou le présent dans la mesure où il ne donne pas à voir le procès dans sa tension.

\subsection{Prospection, ultériorité, futur et relation de progression narrative}

Il est rarement traité du rapport entre les temps qui ouvrent une perspective à venir (présent et imparfait prospectifs, conditionnels, futurs) et la relation de progression narrative. Leur interaction nous paraît pourtant intéressante à analyser.

\subsubsection{Présent et imparfait prospectifs}

Ces deux temps sont formés sur la grammaticalisation de aller. De l'interaction des deux éléments forme itive conjuguée + verbe à l'INF résulte la valeur aspectuelle de prospection, que l'on définira comme orientation 
ascendante de l'actant sujet vers un acte - plus précisément vers la borne initiale de cet acte - représenté par le verbe à l'infinitif (Bres \& Barceló 2007). C'est à partir de cette valeur que peuvent se produire, en interaction avec différents éléments cotextuels, différents effets de sens. Nous ne nous intéresserons ici qu'aux emplois de cette périphrase en textualité narrative, tours dans lesquels elle ne peut se construire qu'au présent et à l'imparfait (Bres 2008).

\subsubsection{Présent prospectif}

L'orientation prospective vers la borne initiale du procès à l'infinitif, si elle s'accorde avec la demande de la relation de progression de prendre en compte la borne initiale, ne la satisfait cependant pas tout à fait : s'orienter vers elle n'est pas forcément l'atteindre. Le mouvement peut toujours être intercepté :

(9) il s'approche d'elle et la regarde dans les yeux. Il va l'embrasser mais on frappe de façon insistante à la porte. Elle se lève d'un bond et demande qui est là. (internet)

Et du coup c'est non seulement la borne initiale qui n'est pas atteinte, mais l'entier du procès qui ne se réalise pas. Or le récit sert principalement à dire ce qui s'est passé... Le présent prospectif ne répond donc que latéralement à la demande de la textualité narrative. Et pourtant... Les langues catalane, française, occitane ont usé, initialement, du présent prospectif comme temps du récit, en alternance avec le présent « historique », le passé composé ou le passé simple, comme dans (10) :

(10) Et estant en ce pensement luy va prendre grant faim de dormir et s'alla fort endormir, et luy dormant se va lever un bon vent pour faire voile (Roman de Pierre de Provence, cité par Gougenhein 1929/1971 : 97).('A cette pensée il lui prend / prit forte envie de dormir et il alla dormir ; pendant q'il dormait, il se lève / s'est levé un bon vent pour naviguer')

Soit la relation de progression [va prendre $<$ s'alla $^{3}$ dormir $<$ se va lever], actualisée par la succession : présent prospectif < passé simple < présent prospectif. Le cotexte lève l'hypothèque précédemment mentionnée : en ne présentant pas cotextuellement d'interception à la prospection de va prendre et de va lever, on comprend - principe de pertinence - que l'envie de dormir a effectivement pris l'actant, et que le bon vent s'est effectivement levé : à savoir qu'ont été atteintes non seulement la borne initiale mais également la borne terminale de ces procès.

Aller a ici sa valeur pleine de verbe de mouvement. 
Ce tour s'est totalement grammaticalisé en catalan dans la mesure où il est devenu un temps du passé, le prétérit périphrastique, qui a actuellement quasiment éliminé le prétérit synthétique. Il en est allé différemment en français : à partir du XVI ${ }^{\mathrm{e}}$ siècle, il disparaît progressivement des textes pour des raisons complexes (Gougenheim 1929/1971), et il n'en est plus fait mention que dans les grammaires historiques. Bien à tort, car il semble qu'il soit parfaitement vivant dans au moins deux genres de textualité narrative : le résumé (11), le reportage « biographique » (12) :

Un lycéen résume oralement Bel-Ami de Maupassant.

Bel-Ami commence au moment où Duroy / un ancien militaire un peu désabusé / marche dans les rues de Paris /à ce moment-là il va rencontrer un ancien camarade / Forestier qui lui a réussi et qui va essayer de l'aider à devenir un gentilhomme [...]

Dans cette ambiance de chasse aux sorcières, il est victime, en 1978, d'une tentative d'assassinat : un tueur raciste lui tire une balle dans le dos. Depuis, Larry Flint a les jambes paralysées. Cloué dans un fauteuil roulant, il va redoubler d'activité, à la fois dans les affaires et en politique. Son plus grand motif de fierté est d'avoir gagné un procès retentissant contre le sénateur [...] (Le Monde, Reportage, Larry Flint contre les hypocrites, 1. 9 2007)

On notera que dans les deux occurrences le temps de base de ces récits est le présent simple : comme lui, le présent prospectif est neutre temporellement ; il actualise les procès de l'événement sans les situer par lui-même dans une époque, à la différence des temps du passé et des temps du futur.

Pour l'heure, l'emploi de ce temps qui, par sa structure aspectuelle, ne répond qu'imparfaitement à la demande de la relation de progression, n'est que sporadique ; et l'on ne peut bien sûr faire des hypothèses sur le sort que lui réservera la langue dans son avenir.

\subsubsection{L'imparfait prospectif}

L'imparfait prospectif présente les mêmes difficultés que le présent : en luimême, il ne fait qu'orienter vers la borne initiale du procès, ce qui laisse toute possibilité d'une interception avant son atteinte :

(13) Il reprit le chemin du grand escalier. Il allait arriver sur le palier du premier étage quand des détonations toutes proches l'obligèrent à s'enfoncer dans une encoignure. (Tournier, Le Roi des Aulnes)

Et il arrive parfois que le procès, d'abord actualisé à ce temps, soit repris au passé simple, pour marquer qu'il a bien eu lieu : 
(14) On avait reçu de bonnes nouvelles de M.. de Beuvre. (...) Il allait arriver ; il arriva, en effet. On lui fit de grandes fêtes. (Sand, Les Beaux Messieurs de Bois-Doré)

De plus, ce temps actualise, en tant qu'imparfait, l'auxiliaire de prospection en non-incidence, ce qui entre également en dissonance avec la demande d'incidence. Malgré ce, du fait de sa valeur prospective, on le trouve en récit sporadiquement, dans deux emplois textuels.

Comme le futur et le conditionnel (cf. infra), il actualise le ou les derniers procès d'une série : à partir du procès précédent le plus souvent au passé simple, il ouvre la perspective sur la conclusion, qu'il contribue de la sorte à mettre en relief :

Le 19 juillet, dans l'étape de la Toussuire, Floyd Landis fut victime d'une terrible défaillance. Il allait franchir la ligne d'arrivée avec plus d'un quart d'heure de retard. (Midi Libre)

D'autre part, et ceci à ma connaissance n'a pas été relevé, dans le discours journalistique racontant un événement sportif ou un fait divers, il alterne avec l'imparfait «narratif »: (les oc. d'imparfait «narratif » sont en italiques, celles d'imparfait prospectif en petites majuscules):

(16) C'est le Tchèque Smicer qui ALLAIT FaIRE basculer la rencontre. A peine entré en jeu, il profitait d'une erreur défensive pour battre Borelli $\left(56^{\mathrm{e}}\right)$. L'exclusion de Lecour $\left(59^{e}\right)$ ALLAIT également FACILITER la tâche des Nordistes. A dix, Caen ALLAIT de nouveau PLIER. Vairelles décalait Smicer qui réalisait le doublet $\left(67^{\mathrm{e}}\right)$. La fin du match ALLAIT ETRE lensoise mais les hommes de Leclerc gâchaient plusieurs occasions de contre. La forte poussée normande, lors des ultimes minutes, n'ALLAIT pas CONNAITRE de réussite. (Midi Libre).

Soulignons la façon dont sont textuellement entrelacées les deux formes, avec d'autant plus de facilité qu'elles actualisent toutes deux un imparfait et qu'elles sont toutes deux associées, de façon latéralement discordante, à la progression, mais de façon différente, dans la mesure où l'imparfait « narratif » la tolère alors que l'imparfait prospectif participe à sa production du fait de son aspect prospectif.

\subsubsection{Le conditionnel}

On analyse le conditionnel, en langue, comme un temps du passé (morphème -ai) qui, à partir d'un énonciateur placé à un point du passé, ouvre une perspective ultérieure (morphème $-r$ ) (Vuillaume 2001). A la différence des autres temps qui en eux-mêmes ne donnent pas l'instruction [+ progression] et à la différence des formes prospectives qui orientent vers la borne initiale 
du procès, le conditionnel pose le procès qu'il actualise comme ultérieur par rapport au point où se situe l'énonciateur, et saisit son temps interne de façon neutre (comme le présent, le futur, etc. : il peut répondre à la demande d'incidence). Il réalise donc par lui-même un mouvement temporel «en avant », et à ce titre, au moins sous sa forme simple, il devrait être un excellent temps narratif. Or tel n'est pas le cas, et ceci pour la raison suivante : le fait que le point à partir duquel est construite l'ultériorité soit un énonciateur passé et non le locuteur actuel entraîne que les procès qui sont envisagés de ce point de vue sont «subjectifs », c'est-à-dire qu'ils ne sont pas inscrits dans la réalité du passé, puisque pour cet énonciateur ces procès sont à venir :

C'était le mois prochain qu'ils devaient s'enfuir. Elle partirait d'Yonville (...), Rodolphe aurait (...) écrit à Paris afin d'avoir la malle entière jusqu'à Marseille, où ils achèteraient une calèche, et, de là, continueraient sans s'arrêter (...). (Flaubert, Madame Bovary)

S'il y a bien progression [partirait < achèteraient < continueraient], c'est une progression imaginée par Emma et non inscrite par le narrateur dans la réalité des faits passés. Or la textualité narrative prétend dire ce qui s'est effectivement passé. Le conditionnel ne sera employé en récit que très secondairement, pour actualiser ce que tel ou tel personnage-énonciateur imagine qu'il se produira, mais pas ce qui s'est effectivement produit.

Il est cependant un autre usage du conditionnel, dit objectif (Haillet 2002): lorsque le locuteur fait comme s'il déléguait sa responsabilité énonciative à un énonciateur passé qui envisage des faits à venir, alors que de fait il raconte des événements dont il sait, à partir de sa position actuelle, qu'ils se sont effectivement produits. Façon stylistique de mettre de la perspective :

(18) Laurent Jalabert portait une attaque rédemptrice dans la descente du col d'Aspin. L'illusion durait quelques kilomètres avant que les sénateurs ne réimposent leur train. Le champion de France paierait plus tard sa folie cher : 1min 14s abandonnée sur la ligne d'arrivée à Jan Ullrich. (fin de l'article, Midi Libre)

Le scripteur de l'article, qui écrit son compte rendu après la fin de l'étape cycliste, sait que l'actant Jalabert a payé «cher sa folie»: on peut parfaitement remplacer le conditionnel par le passé simple (ou tout autre temps du passé effectif) : «Le champion de France paya plus tard sa folie cher». On ne trouve guère ce fonctionnement narratif «objectif» du conditionnel que pour actualiser le ou les derniers procès d'une série. 


\subsubsection{Futur}

Le futur, de par ses instructions aspectuelles [+ tension], [ \pm incidence], répond parfaitement à la demande de la relation de progression, au même titre que le présent. Mais il contrevient, par son instruction [+ futur], à la contrainte temporelle qui n'affecte pas la relation de progression elle-même, mais seulement son fonctionnement en textualité narrative: raconter un événement présuppose que le narrateur connaisse ledit événement. Or on ne peut connaître que le passé, au mieux le présent, mais pas le futur (Bres 2008). De sorte que la forme future ne saurait être le temps de base d'un récit, même s'il se trouve quelques "hapax », comme ce manuscrit édité par E. Le Roy Ladurie \& et O. Ranum, Pierre Prion, scribe, datant du XVIII ${ }^{\mathrm{e}}$ siècle, dans lequel le narrateur raconte sa propre vie comme un « horoscope » :

(19) On le mettra à coucher dans une chambre très reculée ; il sera chargé de la clé d'icelle qu'il aura soin de mettre sous le chevet de son lit. Il sera très étonné le premier matin en se levant de se trouver sans culotte ; il courra à la porte qu'il trouvera bien fermée (...)

Si le futur est extrêmement rare comme temps de base du récit, on le trouve sporadiquement, pour terminer un récit ou un épisode (Labeau 2009), dans le même type d'emploi textuel que le conditionnel ou l'imparfait prospectif :

on apprend que le cimetière sert régulièrement de rencontre nocturne à des jeunes gens de bonne famille. Quarante jeunes sont interpellés. Tous seront finalement relâchés. (Le Monde, août 1996)

Les grammaires, dans ce cas, parlent de futur «narratif » ou « historique ».

Ajoutons que si nous n'avons parlé, pour les temps prospectifs, le conditionnel et le futur, que des formes simples, les formes composées correspondantes sont susceptibles d'actualiser la relation de progression avec les mêmes restrictions que les formes simples, auxquelles vient s'ajouter, comme pour toutes les formes composées, la discordance de leur trait [+ extension].

\section{Conclusion}

Après avoir évoqué quelques possibles écueils dans l'analyse du temps verbal, nous avons présenté rapidement l'analyse que nous faisons des temps de l'indicatif comme système aspectuo-temporel, afin de mettre à l'épreuve son rendement dans l'étude d'une relation de discours - la progression - dans un type de textualité : la textualité narrative. La relation de progression demande que le temps interne des procès soit actualisé (i) en tension, et 
(ii) en incidence. La textualité narrative demande que l'événement soit envisagé dans sa réalisation effective. Il apparaît tout d'abord qu'aucun temps verbal de l'indicatif n'est frontalement allergique à la relation de progression narrative ${ }^{4}$. Mais, si tous les temps peuvent être «narratifs » - à savoir qu'ils peuvent actualiser les procès du premier plan en relation de progression - certains le sont plus que d'autres...

Le passé simple et le présent s'accordent parfaitement, de par leurs instructions aspectuelles, avec la demande aspectuelle de la relation de progression; et de par leur instruction temporelle, avec la demande temporelle de la textualité narrative.

Toutes les autres formes sont en interaction plus ou moins discordante soit avec la demande aspectuelle de la relation de progression, soit avec la demande temporelle de la textualité narrative.

Un premier groupe de temps - imparfait, passé composé, passé antérieur, plus-que-parfait - s'accordent avec la demande temporelle de la textualité narrative, mais sont en désaccord avec tout ou partie de la demande aspectuelle de la relation de progression: l'imparfait ne satisfait pas la demande d'incidence; le passé composé et le passé antérieur, de façon différente, ne satisfont pas la demande de tension; le plus-que-parfait ne satisfait aucune des deux demandes. Cette interaction discordante n'empêche pourtant pas leur usage en contexte de progression narrative; elle rend compte de ce que, à l'exception du passé composé, ledit usage est marginal, et à l'origine d'effets de sens spécifiques.

Un second groupe de temps, qui de différentes façons ouvrent une perspective à venir - présent et imparfait prospectifs, conditionnels, futurs entrent en interaction plus dissonante avec la demande de la progression narrative : par leur structure aspectuelle pour les deux temps prospectifs, par leur instruction temporelle pour le futur et le conditionnel. Ce qui explique qu'ils ne soient que très sporadiquement en emploi « narratif ».

Notons pour finir que le français contemporain ne dispose pas d'un temps verbal parfaitement «narratif » pour raconter un événement passé, à la différence de ce qui se passe p. ex en espagnol avec le prétérit, ou en catalan avec le prétérit périphrastique. Le passé simple se voit réduit au plan d'énonciation de l'histoire, et le présent ne marque pas par lui-même l'époque passée, même s'il peut s'accorder - du fait de son instruction temporelle [+ neutre] - avec elle. C'est peut-être ce qui explique que bien souvent, dans un même récit, on «switche » d'un temps à l'autre, comme dans cette occurrence de compte rendu sportif d'un match de rugby FranceItalie qui mélange allègrement présent, passé simple et passé composé :

Ce qui n'est pas le cas de toutes les relations de discours : le passé simple p. ex. ne peut entrer dans la relation d'inclusion, plus exactement ne peut actualiser le procès inclusif (Bres \& Lauze 2007). 
Michalak à l'envers, c'est alors tout le XV de France qui déjoue. Avant de se reprendre en seconde mi-temps. Attention, les bleus ne furent pas irrésistibles. Simplement, le moral était meilleur, "grâce au public qui a continué à nous encourager », flagorne un Laporte en quête de pardon. Simplement, les jambes de feu de Castaignède et Dominici ont créé des brèches. Simplement, le pack se remit dans l'axe pour, enfin, avoir raison de la résistance italienne. (Le Figaro)

\section{Références}

Asher, N. ; Aurnague, M. ; Bras, M. ; Sablayrolles, P. ; Vieu, L. (1995). De l'espace-temps dans l'analyse du discours, Sémiotiques 9: 11-62.

Barceló, G. J. ; Bres, J. (2006), Les temps de l'indicatif, Paris : Ophrys.

Benveniste, E. (1959 / 1966). Les relations de temps dans le verbe français, in : Problèmes de linguistique générale, Paris : Gallimard, 237-257.

Berthonneau, A.-M. ; Kleiber, G. (2003). Un imparfait de plus... et le train déraillait, Cahiers Chronos $11: 1-24$.

Bres, J. (1998). L'imparfait narratif est un imparfait comme les autres, in : D. Leeman; A. Boone, (eds.), Du percevoir au dire, Mélanges offerts à A. Joly, Paris : L'Harmattan, 261-276.

Bres, J. (2007). Et plus si affinités... Des liaisons entre les instructions du plus-que-parfait et les relations d'ordre temporel, Cahiers Chronos 18 : $139-157$.

Bres, J. (2008). De la production de l'effet de sens grammatical d'imminence-ultériorité : pourquoi peut-on dire le train allait partir, mais non le train *alla partir?, Congrès Mondial de linguistique française, Paris, 9-12 juillet 2008.

Bres, J.; Barceló G. J. (2007). La grammaticalisation de la forme itive comme prospectif dans les langues romanes, in : M. J. Fernandez-Vest, (éd.), Combat pour les langues du monde - Fighting for the world's languages, Hommage à Claude Hagège, Paris, Ed. L'Harmattan, Collection Grammaire \& Cognition, $\mathrm{N}^{\circ} 4$ et 5, 91-103.

Bres, J.; Lauze, A. (2007). La relation d'inclusion et les temps verbaux du passé : point de vue ou aspect?, in: J. Bres; M. Arabyan; Th. Ponchon; L. Rosier ; R. Tremblay; P. Vachon-l'Heureux, (éds), Psychomécanique du langage et linguistiques cognitives, Actes du XIè colloque international de l'AIPL, 8-10 juin 2006, Limoges : Lambert Lucas, 261-270.

Carruthers, J. (2005). Oral Narration in Modern French: A Linguistic Analysis of Temporal Patterns, London : Legenda. 
Gosselin, L. (1996). Sémantique de la temporalité en français, Louvain-la Neuve : Duculot.

Gougenheim, G. (1929/1971). Etude sur les périphrases verbales de la langue française, Paris : Champion.

Guillaume, G. (1929/1970). Temps et verbe, Paris : Champion.

Haillet, P. (2002). Le conditionnel en français. Une approche polyphonique, Paris : Ophrys.

Labeau, E. (2007). Et un, ou deux, ou trois ? Les temps-champions du compte rendu sportif depuis 1950. E. Labeau ; C. Vetters ; P. Caudal, (éds), Sémantique et Diachronie du système verbal français. Amsterdam / New York : Rodopi, 203-233.

Labeau, E. (2009). Le PS, cher disparu de la rubrique nécrologique ?, Journal of French Language Studies, 19/1.

Labov, W. (1978). La transformation du vécu à travers la syntaxe narrative, in : Le parler ordinaire I, Paris : Minuit, 289-355.

Lascarides, A. ; Asher, N. (1993), Temporal Interpretation, Discourse Relations and Commonsense Entailment, Linguistics and Philosophy $16: 437-493$

Majumdar, M.; Morris, A. M. (1980). The French pluperfect tense as a punctual past, Archivum Linguisticum 11.1 : 1-12.

Vuillaume, M. (2001). L'expression du futur dans le passé en français et en allemand, in : P. Dendale ; L. Tasmowski, Le conditionnel en français, Metz : Recherches linguistiques 25, 105-123.

Wilmet, M. (1997). Grammaire critique du français, Paris : Hachette, Louvain-la Neuve : Duculot. 